Clinton B. Wright, MD, MS

Chuanhui Dong, $\mathrm{PhD}$

Matthew Stark, BS

Shonni Silverberg, MD

Tatjana Rundek, MD,

$\mathrm{PhD}$

Mitchell S.V. Elkind,

MD, MS

Ralph L. Sacco, MD, MS

Armando Mendez, PhD

Myles Wolf, MD, MMSc

Correspondence to

Dr. Wright:

c.wright21@med.miami.edu

Supplemental data at Neurology.org

\section{Plasma FGF23 and the risk of stroke}

\author{
The Northern Manhattan Study (NOMAS)
}

ABSTRACT

Objective: To examine fibroblast growth factor 23 (FGF23) as a risk factor for incident stroke in a racially/ethnically diverse population-based urban cohort.

Methods: Stroke-free Northern Manhattan Study participants with FGF23 measurements ( $\mathrm{n}=$ $2,525)$ were followed for a mean of $12( \pm 5)$ years to detect incident strokes. We used Cox proportional hazards models to estimate the association of baseline FGF23 with incident total, ischemic, and hemorrhagic stroke.

Results: Median FGF23 was 57 relative units (RU)/mL (interquartile range $=44-81 \mathrm{RU} / \mathrm{mL}$ ). Each unit increase of natural log-transformed FGF23 conferred a 40\% greater overall stroke risk after adjusting for estimated glomerular filtration rate and sociodemographic and vascular risk factors (hazard ratio $=1.4,95 \%$ confidence interval 1.1-1.6, $p=0.004$ ). Penalized spline analysis revealed a linear association with overall stroke risk at $\geq 90 \mathrm{RU} / \mathrm{mL}$ FGF23, compared with $<90 \mathrm{RU} / \mathrm{mL}$ (hazard ratio $=1.5,95 \%$ confidence interval $=1.2-2.1, p=0.004$ ). Greater FGF23 conferred a doubling of intracerebral hemorrhage $(\mathrm{ICH})$ risk but no significant increased risk of ischemic stroke. The associations of elevated FGF23 levels with greater risks of overall stroke and $\mathrm{ICH}$ events were independent of phosphate and parathyroid hormone levels and were similar among participants without chronic kidney disease.

Conclusions: Elevated FGF23 was a risk factor for overall stroke and ICH events, in particular in a racially and ethnically diverse urban community, independent of chronic kidney disease. Neurology ${ }^{\circledR}$ 2014;82:1700-1706

\section{GLOSSARY}

$\mathbf{C I}=$ confidence interval; $\mathbf{C K D}=$ chronic kidney disease; $\mathbf{C V}$ = coefficient of variation; eGFR = estimated glomerular filtration rate; FGF23 = fibroblast growth factor 23; HR = hazard ratio; ICD-9 = International Classification of Diseases, ninth revision; $\mathbf{I C H}=$ intracerebral hemorrhage; $\mathbf{L V H}=$ left ventricular hypertrophy; NOMAS = Northern Manhattan Study; PTH = parathyroid hormone; $\mathbf{R U}=$ relative units; $\mathbf{S A H}=$ subarachnoid hemorrhage.

Identification of novel stroke risk factors is of great public health importance. Fibroblast growth factor 23 (FGF23) is a hormone secreted by osteocytes that regulates phosphate homeostasis through effects on the proximal renal tubule by inducing phosphaturia and inhibiting calcitriol production. Elevated FGF23 levels are the earliest marker of disordered mineral metabolism in chronic kidney disease (CKD), beginning as early as stage 2 (estimated glomerular filtration rate [eGFR] 60-89 mL/min $/ 1.73 \mathrm{~m}^{2}$ ), and are strongly associated with mortality across the spectrum of CKD severity and in the general population without CKD. ${ }^{1-3}$ Elevated FGF23 is linked with altered cardiac structure and function and atherosclerosis, although the mechanisms are poorly understood. ${ }^{4-7}$

Elevated FGF23 has been linked with a greater risk of cardiovascular mortality and combined vascular outcomes that included stroke, but few studies have examined stroke separately. ${ }^{8,9}$ In people with established heart disease, those with elevated FGF23 had an increased risk of stroke, but data from population-based studies that include people without established heart disease or

From the Evelyn F. McKnight Brain Institute (C.B.W., C.D., T.R., R.L.S.) and Departments of Neurology (C.B.W., C.D., M.S., T.R., R.L.S.), Public Health Sciences (C.B.W., R.L.S.), Human Genomics (R.L.S.), and Medicine (A.M., M.W.), Leonard M. Miller School of Medicine, and the Neuroscience Program (C.B.W., R.L.S.), University of Miami, FL; and Departments of Neurology (M.S.V.E.) and Medicine (S.S.), College of Physicians and Surgeons, and Department of Epidemiology (M.S.V.E.), Mailman School of Public Health, Columbia University, New York. Go to Neurology.org for full disclosures. Funding information and disclosures deemed relevant by the authors, if any, are provided at the end of the article. 
CKD are limited and conflicted. ${ }^{10}$ In one community-based study, participants with elevated FGF23 had greater stroke risk, but only in those with $\mathrm{CKD} .^{3}$ Also, data are lacking on FGF23 as a risk factor for stroke among Hispanic/Latino people who are at greater risk of stroke than white people. ${ }^{11}$

We report on plasma FGF23 in the racially/ ethnically diverse Northern Manhattan Study (NOMAS) to test our hypothesis that elevated FGF23 is an independent risk factor for incident stroke in this urban, community-based population.

METHODS Study population. The NOMAS was designed to determine stroke incidence, risk factors, and prognosis in a racially/ethnically diverse urban population. Study details have been published previously. ${ }^{12}$ Briefly, eligible participants were (1) stroke-free, (2) older than 40 years, and (3) residents of Northern Manhattan for at least 3 months in a household with a telephone. Audits and Surveys, Inc., performed random digit dialing using dual-frame sampling (telephone response rate was 91\%) and participants were invited to enroll with an in-person interview and neurologic assessment (enrollment response rate was $75 \%$ ). The overall participation rate was $69 \%$, and a total of 3,298 subjects were enrolled between 1993 and 2001.

Standard protocol approvals, registrations, and patient consents. All participants signed written informed consent and the institutional review boards of Columbia University Medical Center and the University of Miami approved the study.

Baseline evaluation. Trained research assistants collected data through interviews in English or Spanish, depending on the language spoken at home. Study physicians performed physical examinations. Race and ethnicity were determined based on selfidentification using questionnaires modeled after the US census. ${ }^{13}$ Standardized questions regarding hypertension, diabetes mellitus, smoking, and cardiac conditions were adapted from the Behavioral Risk Factor Surveillance System by the Centers for Disease Control. ${ }^{14}$ Measurement of blood pressure and fasting blood specimens for glucose and lipids have been described. ${ }^{15}$

Processing of blood samples and assays. Baseline fasting blood specimens were spun immediately at $-4^{\circ} \mathrm{C}$ and stored at $-70^{\circ} \mathrm{C}$ until processing. Plasma C-terminal FGF23 concentrations were measured in duplicate using second-generation ELISA (Immutopics International, San Clemente, CA). The intra-assay coefficient of variation $(\mathrm{CV})$ was 3.6 ; the interassay $\mathrm{CVs}$ evaluated with quality-control samples from 26 independent assay runs were $2.7 \%$ at a concentration of 31 relative units $(\mathrm{RU}) / \mathrm{mL}$ and $4.4 \%$ at a level $272 \mathrm{RU} / \mathrm{mL}$. Serum phosphate, intact (1-84) parathyroid hormone (PTH), and cystatin C were measured in duplicate on a Roche Cobas 6000 analyzer, using the manufacturer's reagents (Roche Diagnostics, Indianapolis, IN). Serum phosphate was measured spectrophotometrically with intraand interassay $\mathrm{CVs}$ of less than $3 \%$ (reference range $2.5-4.5 \mathrm{mg} / \mathrm{dL}$ ). PTH was measured by electrochemiluminescence immunoassay with an intra-assay $\mathrm{CV}$ of $2.8 \%$, and an interassay $\mathrm{CV}$ of $6.1 \%$ (reference range $15-65 \mathrm{pg} / \mathrm{mL}$ ). Serum creatinine was measured using the kinetic alkaline picrate assay (Jaffé reaction). Cystatin C was measured using particle-enhanced immunoturbidimetric assay with aggregates determined spectrophotometrically at $546 \mathrm{~nm}$, with an intra-assay CV of $2.8 \%$ and an interassay $\mathrm{CV}$ of $4.1 \%$ (reference range $0.5-1.3 \mathrm{mg} / \mathrm{L}$ ).

Outcomes. The primary outcome was adjudicated incident stroke. Follow-up procedures and outcome classifications have been published. ${ }^{15}$ Briefly, participants and/or family members are interviewed annually by telephone to determine changes in vital status, detect neurologic events, and document interval hospitalizations. The outcome surveillance network includes daily screening of admissions, review of neurology consult lists with covering house staff, hospital admission and discharge data (including screening of $I C D$-9 codes), emergency room visits, and visits to the ambulatory care network. The average annual contact rate has been $99 \%$, with only $1 \%$ lost to follow-up. A specially trained research assistant reviews all stroke events, and medical records of all hospitalizations are reviewed to verify details of suspected events. Persons who screen positive undergo in-person assessment, chart review, and examination by a study neurologist. Stroke events were classified as the first occurrence of ischemic stroke, intracerebral hemorrhage (ICH), subarachnoid hemorrhage $(\mathrm{SAH})$, and unknown stroke type. Two NOMAS vascular neurologists adjudicated each stroke case independently, and a third resolved disagreements.

Statistical analysis. The current study was limited to participants with available baseline FGF23 and creatinine measurements $\left(\mathrm{n}=2,525\right.$; figure e-1 on the Neurology ${ }^{\circledR}$ Web site at Neurology.org). All covariates were also measured at baseline. For our primary analysis, eGFR was defined using the Modification of Diet in Renal Disease formula as: GFR $=186$. $3 \times($ serum $\mathrm{Cr})-1.154 \times$ age $-0.203 \times(0.742$ if female $) \times$ (1.21 if black). ${ }^{16}$ We confirmed our findings using several alternative eGFR formulas, including the Chronic Kidney Disease Epidemiology equation, those based on cystatin C, and the combination of the $2 .{ }^{17,18}$ Values for FGF23 and PTH were natural $\log$-transformed to achieve normal distributions.

We used Cox proportional hazards models to estimate the risk conferred by plasma FGF23 levels on incident stroke, overall and by ischemic and hemorrhagic stroke subtypes. We used penalized spline analysis to determine whether the association between FGF23 and incident stroke was nonlinear. We then examined natural log-transformed FGF23 as a continuous variable, and created a binary variable based on a cut-point defined by the penalized spline analysis. Person-time of follow-up accrued from baseline to the last follow-up, the time of incident stroke, death, or loss to follow-up, whichever came first. We constructed 3 sequential models. Model 1 was adjusted for age, sex, race, ethnicity, and eGFR. Model 2 was adjusted further for smoking, reported alcohol use, body mass index, a history of cardiac disease, fasting cholesterol, cholesterollowering medication use, fasting glucose, glycemic medication use, systolic and diastolic blood pressures, and antihypertensive use. Model 3 was adjusted further for serum phosphate and PTH. We ran our models in those without evidence of CKD (eGFR $60 \mathrm{~mL} / \mathrm{min} / 1.73 \mathrm{~m}^{2}$ ) and did sensitivity analyses to (1) exclude those with possible primary hyperparathyroidism, and (2) confirm our findings using the alternative methods to estimate GFR. All tests were 2-tailed, and a $p$ value $<0.05$ was considered significant. Spline analysis was conducted using smoothHR (R Project, www.r-project.org) and other analyses were conducted using SAS version 9.3 (SAS Institute, Cary, NC).

RESULTS There were 2,525 NOMAS participants with FGF23 and creatinine measurements. There 
Table 1 Northern Manhattan Study: Sample characteristics

\begin{tabular}{|c|c|c|c|}
\hline Characteristic & Total & FGF23 $<90$ & FGF23 $\geq 90$ \\
\hline FGF23 range, $\mathrm{RU} / \mathrm{mL}$ & $6.6-1,389.9$ & $6.6-89.9$ & $90.0-1,389.9$ \\
\hline Sample size & 2,525 & 2,020 & 505 \\
\hline Age, y, mean (SD) & $69(10)$ & $68(10)$ & $72(10)$ \\
\hline \multicolumn{4}{|l|}{ Sex, n (\%) } \\
\hline Male & $919(36)$ & 771 (38) & $148(29)$ \\
\hline Female & $1,606(64)$ & $1,249(62)$ & $357(71)$ \\
\hline \multicolumn{4}{|l|}{ Race/ethnicity, n (\%) } \\
\hline Hispanic & $1,353(54)$ & $1,108(55)$ & $245(49)$ \\
\hline Black & $590(23)$ & $439(22)$ & $151(30)$ \\
\hline White & $521(21)$ & 425 (21) & $96(19)$ \\
\hline Other & $61(2)$ & $48(2)$ & $13(2)$ \\
\hline Current smoker, n (\%) & $426(17)$ & $323(16)$ & $103(20)$ \\
\hline Moderate ethanol, n (\%) & $866(34)$ & 724 (36) & $142(28)$ \\
\hline \multicolumn{4}{|l|}{ Medications, n (\%) } \\
\hline Antihypertensives & $1,127(45)$ & $836(41)$ & $291(58)$ \\
\hline Glycemic agents & 365 (15) & $261(13)$ & $104(21)$ \\
\hline Cholesterol-lowering & $358(14)$ & $269(13)$ & $89(18)$ \\
\hline Diastolic BP, mm Hg, mean (SD) & $83(11)$ & $83(11)$ & $84(12)$ \\
\hline Systolic BP, mm Hg, mean (SD) & $144(21)$ & $144(21)$ & $147(22)$ \\
\hline Fasting glucose, mg/dL, mean (SD) & $105(47)$ & $103(45)$ & $111(54)$ \\
\hline Total cholesterol, mg/dL, mean (SD) & $203(40)$ & $204(40)$ & $200(43)$ \\
\hline BMI, kg/m², mean (SD) & $28(5)$ & $28(5)$ & $28(6)$ \\
\hline \multicolumn{4}{|l|}{ Biomarkers } \\
\hline FGF23, RU/mL, mean (SD) ${ }^{a}$ & $91(143)$ & $53(16)$ & $241(271)$ \\
\hline Phosphate, mg/dL, mean (SD) & $3(0.5)$ & $3(0.5)$ & $3(0.6)$ \\
\hline PTH, pg/mL, mean (SD) ${ }^{\mathrm{a}}$ & $59(41)$ & $55(23)$ & $76(77)$ \\
\hline eGFR, mL/min/1.73 $\mathrm{m}^{2}$, mean (SD) & $80(22)$ & $82(21)$ & $72(27)$ \\
\hline Creatinine, mg/dL & $1.0(0.4)$ & $0.9(0.3)$ & $1.1(0.7)$ \\
\hline Cystatin $\mathrm{C}, \mathrm{mg} / \mathrm{dL}$ & $1.4(0.6)$ & $1.3(0.3)$ & $1.8(1.0)$ \\
\hline
\end{tabular}

Abbreviations: $\mathrm{BMI}=$ body mass index; $\mathrm{BP}=$ blood pressure; eGFR = estimated glomerular filtration rate; FGF23 = fibroblast growth factor 23; PTH = parathyroid hormone; RU = relative units.

${ }^{a}$ Natural log-transformed for statistical analyses.

were minor differences among participants in the study sample and those not included (table e-1). Sample characteristics are presented in table 1 , overall and based on a spline-derived FGF23 cutoff of $90 \mathrm{RU} / \mathrm{mL}$ (described below). Demographics were similar to the complete NOMAS cohort, with a mean age of almost 70 years and more women and people of Hispanic/Latino origin. The median FGF23 was $57 \mathrm{RU} / \mathrm{mL}$ (interquartile range $=44-81 \mathrm{RU} / \mathrm{mL}$ ). The correlations of $\log$ transformed FGF23 with phosphate, calcium, PTH, and eGFR were weak (table e-2).

Over a mean follow-up of $12( \pm 5)$ years, 258 participants had incident strokes. Elevated FGF23, expressed as a continuous variable, was associated with a greater risk of incident stroke after adjustment for sociodemographic factors, eGFR, and modifiable vascular risk factors (table 2). After adjusting further for PTH and phosphate, the risk was slightly attenuated but remained significantly increased.

Penalized spline analysis adjusted for age showed that log-transformed FGF23 increased incident stroke risk significantly in a linear manner (figure 1). The penalized spline analysis revealed that participants with FGF23 levels more than approximately $90 \mathrm{RU} / \mathrm{mL}$, corresponding to the 80th percentile, had elevated stroke risk. We therefore created a binary variable using a $90 \mathrm{RU} / \mathrm{mL}$ cutoff. A Kaplan-Meier analysis is shown in figure 2A. In a multivariable model adjusting for demographic factors and eGFR, participants with FGF23 values of $\geq 90 \mathrm{RU} / \mathrm{mL}$ had a $60 \%$ greater risk 


\begin{tabular}{|c|c|c|c|c|c|}
\hline \multicolumn{6}{|c|}{ Northern Manhattan Study: Multivariable models of FGF23 and stroke risk } \\
\hline Model & $\begin{array}{l}\text { Continuous: HR }(95 \% \mathrm{Cl}) \\
\text { per unit In-FGF23 }\end{array}$ & p Value & $\begin{array}{l}\text { FGF23 <90 RU/mL, } \\
\text { HR }(95 \% \mathrm{Cl})\end{array}$ & $\begin{array}{l}\text { FGF23 } \geq 90 \mathrm{RU} / \mathrm{mL} \text {, } \\
\text { HR }(95 \% \mathrm{Cl})\end{array}$ & p Value \\
\hline \multicolumn{6}{|l|}{ Any stroke } \\
\hline Rate $(n) / 1,000$ person-years & $9.1(258)$ & & $8.0(191)$ & $14.9(67)$ & \\
\hline Model 1 & $1.4(1.2-1.7)$ & $<0.001$ & 1.0 (reference) & $1.6(1.2-2.2)$ & 0.001 \\
\hline Model 2 & $1.3(1.1-1.6)$ & 0.005 & 1.0 (reference) & $1.5(1.1-2.0)$ & 0.011 \\
\hline Model 3 & $1.3(1.1-1.6)$ & 0.015 & 1.0 (reference) & $1.4(1.0-1.9)$ & 0.028 \\
\hline \multicolumn{6}{|l|}{ Ischemic stroke } \\
\hline Rate $(n) / 1,000$ person-years & 7.5 (212) & & $6.8(162)$ & $11.1(50)$ & \\
\hline Model 1 & $1.2(0.9-1.5)$ & 0.129 & 1.0 (reference) & $1.4(1.0-1.9)$ & 0.053 \\
\hline Model 2 & $1.1(0.9-1.4)$ & 0.498 & 1.0 (reference) & $1.3(1.0-1.9)$ & 0.172 \\
\hline Model 3 & $1.1(0.8-1.3)$ & 0.645 & 1.0 (reference) & $1.2(0.9-1.7)$ & 0.237 \\
\hline \multicolumn{6}{|l|}{ Intracerebral hemorrhage } \\
\hline Rate $(n) / 1,000$ person-years & $0.9(26)$ & & $0.7(16)$ & $2.2(10)$ & \\
\hline Model 1 & $2.2(1.5-3.4)$ & $<0.001$ & 1.0 (reference) & $2.6(1.1-6.1)$ & 0.026 \\
\hline Model 2 & $2.2(1.5-3.4)$ & $<0.001$ & 1.0 (reference) & $2.4(1.0-5.7)$ & 0.049 \\
\hline Model 3 & $2.1(1.3-3.5)$ & 0.003 & 1.0 (reference) & $2.1(0.8-5.2)$ & 0.122 \\
\hline
\end{tabular}

Abbreviations: $\mathrm{BMI}=$ body mass index; $\mathrm{Cl}=$ confidence interval; FGF23 = fibroblast growth factor 23; $\mathrm{HR}=$ hazard ratio; $\mathrm{RU}=$ relative units. Model 1: adjusted for age, sex, race/ethnicity, and estimated glomerular filtration rate. Model 2: covariates in model 1 plus systolic and diastolic blood pressure, antihypertensive medication use, fasting glucose, use of glycemic medications or insulin, fasting total cholesterol, lipid-lowering medication use, current or past tobacco use, moderate alcohol use (one ethanol drink per month to 2 drinks per day, compared with other categories), and BMI. Model 3: covariates in model 2 plus phosphate and parathyroid hormone.

${ }^{\mathrm{a}}$ In = natural log-transformed.

of stroke than those with values $<90 \mathrm{RU} / \mathrm{mL}$, and the risk was attenuated only slightly after adjusting further for vascular risk factors and for phosphate and PTH (table 2). The effect of FGF23 on stroke risk did not differ by race/ethnicity.

Figure 1 Northern Manhattan Study: Penalized spline of association between FGF23 and stroke risk

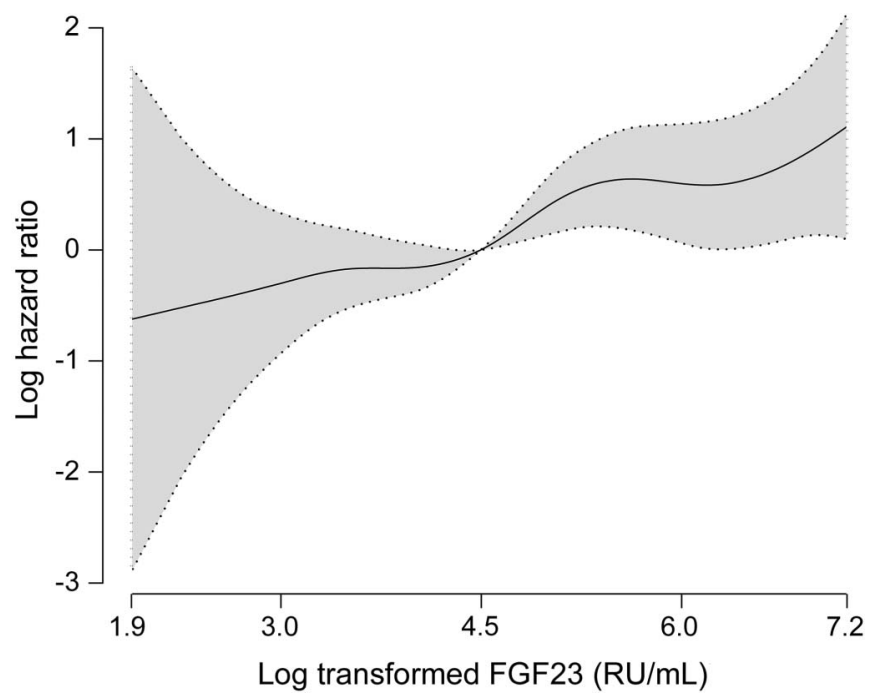

Penalized spline showing the linear association $\left(\chi^{2}=16, p=0.00005\right)$ between natural logtransformed fibroblast growth factor 23 (FGF23) and stroke risk, adjusted for age. The nonlinear term was not significant $\left(x^{2}=4.3, p=0.24\right)$. FGF23 was associated with stroke above a log-transformed level of 4.5 relative units (RU) per $\mathrm{mL}$, equivalent to $90 \mathrm{RU} / \mathrm{mL}$.
Of 258 strokes, 212 were ischemic, 26 were ICHs, 5 were SAHs, and 15 were of unknown type (table e-2). Kaplan-Meier curves for FGF23 and both ischemic stroke and ICH are shown in figure 2, B and C. We found that participants with FGF23 $\geq 90 \mathrm{RU} / \mathrm{mL}$ had marginally more incident ischemic strokes than those with FGF23 $<90 \mathrm{RU} / \mathrm{mL}$ after adjusting for sociodemographic factors and eGFR, but this was no longer significant after further adjusting for vascular risk factors, or phosphate and PTH (table 2). However, we found a doubling in the risk of $\mathrm{ICH}$ per each unit increase in natural log-transformed FGF23 after adjusting for sociodemographic factors and eGFR that remained significant after further adjusting for vascular risk factors, phosphate, and PTH. Participants with an FGF23 of $\geq 90$ vs $<90 \mathrm{RU} / \mathrm{mL}$ also had a significantly greater risk of $\mathrm{ICH}$ adjusting for sociodemographic, eGFR, and vascular risk factors, but this was no longer significant after adjusting for phosphate and PTH (table 2). In a post hoc analysis, we examined FGF23 as a predictor of incident lacunar stroke, but elevated FGF23 did not confer increased risk of lacunar stroke.

Testing for effect modifiers of the risk of stroke conferred by elevated FGF23, we found a marginal interaction for blood pressure such that the effect of FGF23 on ICH risk was stronger among those with higher diastolic blood pressures $(p$ for interaction $=$ 0.06). We found no interaction between FGF23 and 
Figure 2 Northern Manhattan Study: Kaplan-Meier survival curves of FGF23 and stroke risk

A

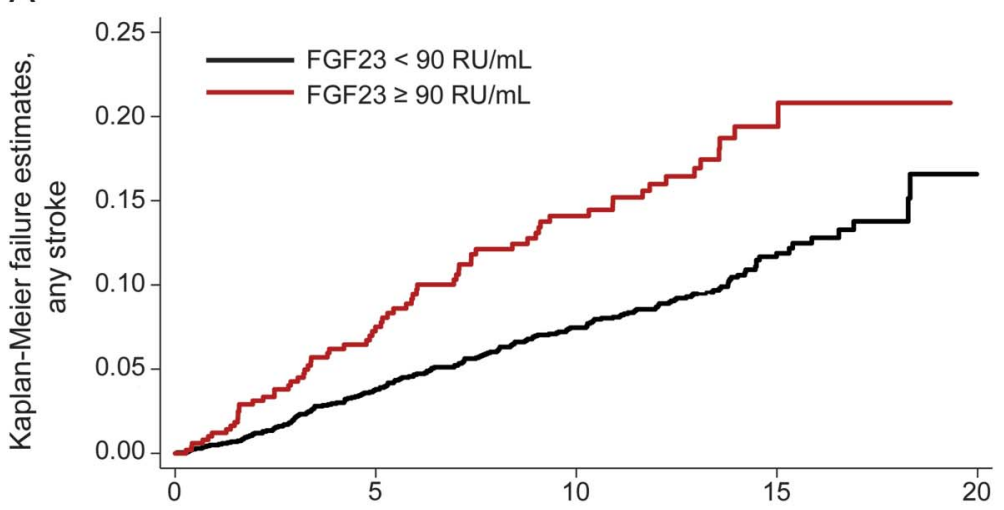

B
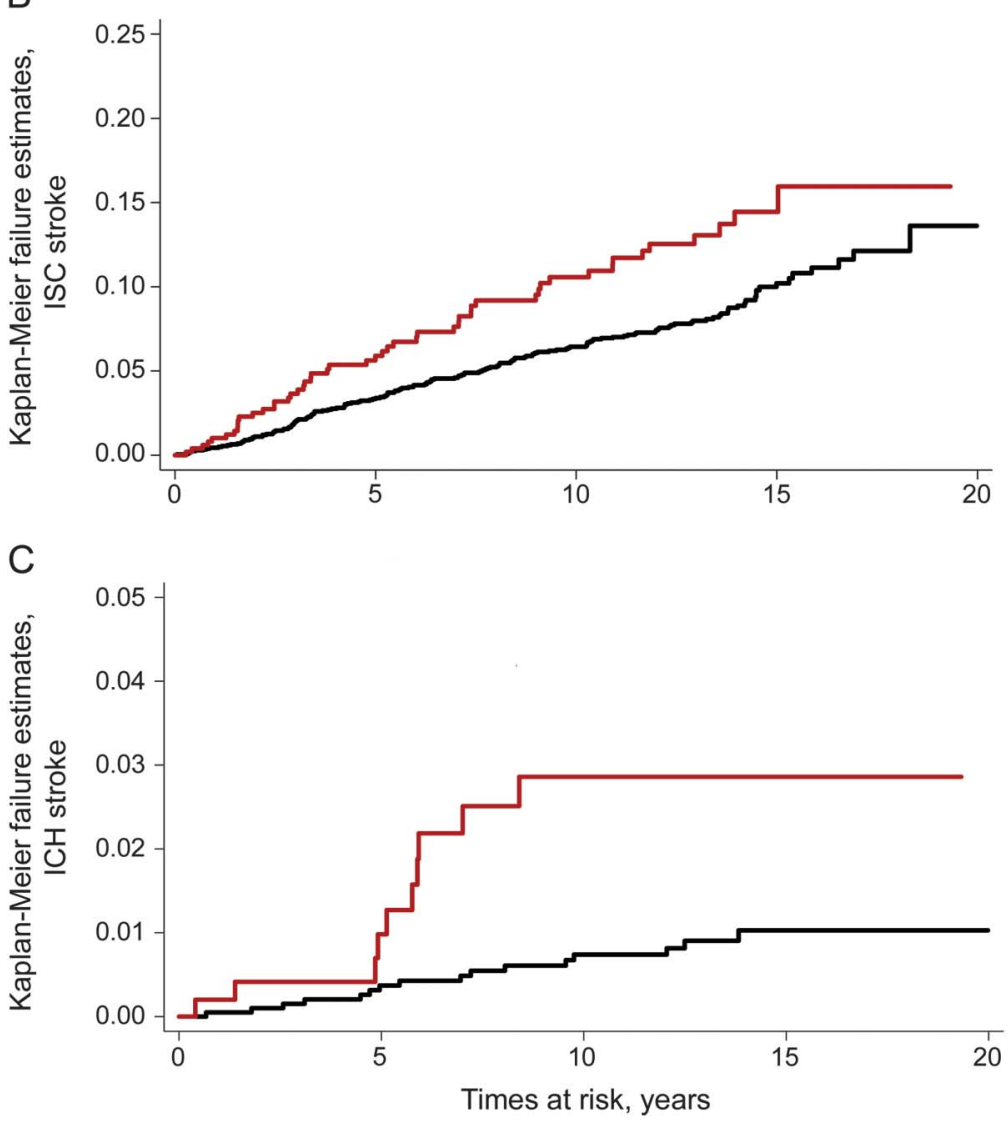

Kaplan-Meier curves comparing participants with fibroblast growth factor 23 (FGF23) levels of $\geq 90$ relative units $(R U) / m L$ with participants with FGF23 levels $<90 \mathrm{RU} / \mathrm{mL}$ for overall stroke (A), ischemic stroke (ISC) (B), and intracerebral hemorrhage (ICH) (C).

race/ethnicity or other risk factors. Only 5 incident $\mathrm{SAH}$ events occurred in our cohort during follow-up, and SAH was not significantly more common in those with elevated FGF23.

In a prespecified analysis to examine the effect of FGF23 on incident stroke in participants without evidence of CKD, we examined people with an eGFR of $\geq 60 \mathrm{~mL} / \mathrm{min} / 1.73 \mathrm{~m}^{2}(\mathrm{n}=2,112)$. Each unit increase in natural log-transformed FGF23 conferred a greater risk of overall stroke (hazard ratio [HR] for incident stroke $=1.4,95 \%$ confidence interval $[\mathrm{CI}]=1.1-1.7, p=0.008)$ and ICH (HR for incident $\mathrm{ICH}=2.2,95 \% \mathrm{CI}=1.2-4.1, p=0.009)$, but not ischemic stroke (HR for incident ischemic stroke $=1.2,95 \% \mathrm{CI}=0.9-1.5, p=0.227)$, even after adjusting for eGFR continuously, and sociodemographic and vascular risk factors.

We did several sensitivity analyses to confirm the robustness of our findings. To determine whether primary hyperparathyroidism might partly explain the increased risk of stroke conferred by elevated FGF23, we excluded those with both abnormally high PTH $(>65 \mathrm{pg} / \mathrm{mL})$ and serum calcium levels $(>10.3 \mathrm{mg} /$ $\mathrm{dL} ; \mathrm{n}=9$ for total stroke, $\mathrm{n}=6$ for ICH). Those with greater FGF23 still had significantly more strokes overall (HR per unit increase in natural log-transformed FGF23 $=1.4,95 \% \mathrm{CI}=1.1-1.6)$ and more ICHs (HR per unit increase in natural log-transformed FGF23 $=2.2,95 \% \mathrm{CI}=1.5-3.4)$. When we estimated GFR using alternative formulas based on creatinine, cystatin $\mathrm{C}$, or the combination of the 2 markers, the findings regarding log-transformed FGF23 and both overall stroke and $\mathrm{ICH}$ risk were similar (data not shown).

DISCUSSION In this population-based urban sample, we found that participants with higher FGF23 levels had a greater risk of incident stroke, in particular ICH. We also found that elevated FGF23 conferred greater stroke risk among those without evidence of CKD.

In a clinic-based sample, those with elevated FGF23 had greater risk of stroke or TIA, but all participants had stable cardiovascular disease. ${ }^{10}$ The populationbased Cardiovascular Health Study found that participants with elevated FGF23 had greater stroke risk, but the effect was limited to those with an eGFR $<60 \mathrm{~mL} /$ $\min / 1.73 \mathrm{~m}^{2}$ or a urine albumin to creatinine ratio $>30 \mathrm{mg} / \mathrm{g}$, suggestive of CKD. ${ }^{3}$ Given that there were fewer people with a low eGFR who had incident strokes in NOMAS, we may have lacked power to detect a statistically significant effect in this group.

Our finding that participants with greater FGF23 levels had more incident strokes driven primarily by ICH risk was unexpected. The mechanisms underlying the risk conferred by FGF23 of vascular outcomes are not completely understood. Adjusting for traditional modifiable risk factors, including blood pressure, attenuated the risk of incident stroke conferred by greater FGF23 only slightly, suggesting some independence from these potential mediators. However, hypertension is a potent modifiable risk factor for $\mathrm{ICH}$, and the synergy between diastolic blood pressure and FGF23 levels may partially explain the increased risk of $\mathrm{ICH}$. While hypertensive ICH and lacunar stroke both result from microvascular damage, FGF23 did not increase the risk of lacunar stroke. Baseline blood 
pressures were higher among those who developed $\mathrm{ICH}$ than those who developed lacunar strokes (data not shown), perhaps partly explaining these findings.

Direct hormonal effects on the vascular system provide plausible mechanisms by which FGF23 may increase stroke risk. In advanced CKD, elevated FGF23 may lead to impaired flow-mediated dilatation of vessels, a marker of endothelial dysfunction related to atherosclerosis, and improvements in flowmediated dilatation after transplant have correlated with decreasing FGF23 levels. ${ }^{19}$ Elevations of FGF23 have been linked with systemic atherosclerosis, and aortic, coronary, and carotid artery calcification across a range of CKD stages, and with aortic calcification in normal men, but these findings require further confirmation. ${ }^{20-22}$ Recent population-based data in humans have also shown that people with elevated FGF23 have a greater prevalence and incidence of left ventricular hypertrophy (LVH), and a study in rodents suggested a causal role: FGF23 caused hypertrophy of cultured myocytes and rat left ventricle, independent of blood pressure, and molecules that block FGF receptors prevented $\mathrm{LVH}^{7,22} \mathrm{LVH}$, increased arterial stiffness, and atherosclerosis are interrelated and could partially explain the effect of FGF23 on stroke risk. If FGF23 contributes to vessel fragility, this might help explain the increased risk of $\mathrm{ICH}$ found here.

The role of altered phosphorus metabolism in vascular damage and resulting outcomes, especially in people without $\mathrm{CKD}$, is unclear. In this population-based sample, the effect of FGF23 on overall stroke and ICH risk was seen in those with good kidney function, and serum phosphate levels were mostly in the normal range. This is not surprising: even in CKD, elevated FGF23 precedes hyperphosphatemia, and in advanced CKD, those with elevated FGF23 have greater mortality even with normal serum phosphate levels. ${ }^{1}$ Why FGF23 is elevated in some with normal kidney function remains unclear, but may be a consequence of unknown genetic factors and increased dietary phosphorus intake. Inorganic phosphate is a major constituent of prepared foods (although reporting on food labels is not required) and has high bioavailability, providing a possible explanation for the finding of higher FGF23 levels in those with low income and education. ${ }^{2}$ PTH itself has cardiovascular consequences, and those with higher PTH levels have been found to have $\mathrm{LVH}$, although studies have generally not adjusted for FGF23. ${ }^{23,24}$

Greater FGF23 levels increased overall stroke and ICH risk after adjusting for phosphate and PTH levels, making it unlikely that these other mineral metabolites mediate our findings. However, we found that PTH levels were greater in those with FGF23 levels $\geq 90$ $\mathrm{RU} / \mathrm{mL}$ (table 1). Primary hyperparathyroidism is unlikely to explain the finding, because it has a relatively low prevalence in normal populations, and excluding people with both high PTH and calcium levels did not alter our findings. ${ }^{25}$

Several limitations are noteworthy. Similar to other studies that examined FGF23 as a predictor of vascular outcomes, because only one baseline measurement of FGF23 was available, we could not account for changes in FGF23 over time. However, we have reported little within-individual variability over time, ${ }^{26}$ and greater variability would tend to bias our results toward the null. We lacked data on dietary phosphorus intake or urinary phosphate excretion as a surrogate of net phosphate exposure. Also, we were unable to process FGF23 in 735 NOMAS participants without plasma available, but those included were slightly healthier than the overall sample and this would tend to prejudice against our finding that elevated FGF23 confers an increased risk of stroke. People with low 25-hydroxyvitamin D, which can lead to secondary hyperparathyroidism, have been found to have an increased risk of stroke in a metaanalysis, but these studies did not adjust for FGF23. ${ }^{27}$ Low 1,25-hydroxyvitamin D in response to elevated FGF23 could be a mediator, a finding supported by animal models of early CKD, but vitamin $\mathrm{D}$ levels were not available for this study. ${ }^{28}$ The strengths of our study include its population-based prospective design, the racially/ethnically diverse urban population, and the lengthy observation with few people lost to follow-up. Our carefully adjudicated stroke outcomes, comprehensive collection of vascular and other behavioral/lifestyle risk factors, and the ability to adjust for important components of mineral metabolism are strengths. Nonetheless, residual confounding is a potential limitation of observational studies such as this one.

Our study provides evidence that relative elevations of FGF23 confer increased risk of stroke, in particular $\mathrm{ICH}$, independent of $\mathrm{CKD}$. Our racially/ethnically diverse community-based sample provides data applicable to a US urban population with a large proportion of Hispanic/Latino individuals. Data from other cohorts are needed to determine whether these findings generalize to other groups. The mechanisms underlying our findings and the roles of components of the mineral metabolism pathway require further study, but if a direct effect is established between FGF23 and stroke risk, it could have treatment implications for dietary and pharmacologic interventions to lower FGF23.

\section{AUTHOR CONTRIBUTIONS}

Dr. Wright: acquisition of data, drafting/revising the manuscript for content, study concept and design, analysis or interpretation of data, study supervision, obtaining funding. Dr. Dong: statistical analysis, drafting/ revising the manuscript for content, analysis or interpretation of data. Mr. Stark: drafting/revising the manuscript for content. Dr. Silverberg and Dr. Rundek: drafting/revising the manuscript for content, analysis and interpretation of data. Dr. Elkind and Dr. Sacco: acquisition of data, drafting/revising the manuscript for content, analysis and interpretation of data, obtaining funding. Dr. Mendez: acquisition of data, study 
supervision, analysis and interpretation of data, drafting/revising the manuscript for content. Dr. Wolf: study concept and design, analysis and interpretation of data, drafting/revising the manuscript for content.

\section{ACKNOWLEDGMENT}

The authors thank Kenneth Cheung, PhD, for biostatistics advice, as well as the NOMAS staff, especially Janet DeRosa, the project manager.

\section{STUDY FUNDING}

Sponsored by the National Heart Lung and Blood Institute (R01 HL 108623) and the National Institute of Neurological Disorders and Stroke (R37 NS 29993).

\section{DISCLOSURE}

C. Wright is funded by related grants from the NIH (K02 NS 059729, R01 HL 108623, R37 NS 29998). C. Dong is funded by related grants from the NIH (R01 HL 108623, R37 NS 29998). M. Stark reports no disclosures relevant to the manuscript. S. Silverberg is funded by a related grant from the NIH (R01 HL 108623). T. Rundek is funded by related grants from the NIH (R01 HL 108623, R37 NS 29998). M. Elkind is funded by related grants from the NIH (R01 HL 108623, R37 NS 29998). R. Sacco is funded by related grants from the NIH (R01 HL 108623, R37 NS 29998). A. Mendez is funded by a related grant from the NIH (R01 HL 108623). M. Wolf is funded by related grants from the NIH (R01 HL 108623, R01DK076116, R01DK081374, R01DK094796, K24DK093723, and U01DK099930). Go to Neurology.org for full disclosures.

Received September 25, 2013. Accepted in final form February 6, 2014.

\section{REFERENCES}

1. Gutierrez OM, Mannstadt M, Isakova T, et al. Fibroblast growth factor 23 and mortality among patients undergoing hemodialysis. N Engl J Med 2008;359:584-592.

2. Isakova T, Xie H, Yang W, et al. Fibroblast growth factor 23 and risks of mortality and end-stage renal disease in patients with chronic kidney disease. JAMA 2011;305:2432-2439.

3. Ix JH, Katz R, Kestenbaum BR, et al. Fibroblast growth factor-23 and death, heart failure, and cardiovascular events in community-living individuals: CHS (Cardiovascular Health Study). J Am Coll Cardiol 2012;60:200-207.

4. Schoppet M, Hofbauer LC, Brinskelle-Schmal N, et al. Serum level of the phosphaturic factor FGF23 is associated with abdominal aortic calcification in men: the STRAMBO Study. J Clin Endocrinol Metab 2012;97:E575-E583.

5. Lim K, Lu TS, Molostvov G, et al. Vascular Klotho deficiency potentiates the development of human artery calcification and mediates resistance to fibroblast growth factor 23. Circulation 2012;125:2243-2255.

6. Gutierrez OM, Januzzi JL, Isakova T, et al. Fibroblast growth factor 23 and left ventricular hypertrophy in chronic kidney disease. Circulation 2009;119:2545-2552.

7. Faul C, Amaral AP, Oskouei B, et al. FGF23 induces left ventricular hypertrophy. J Clin Invest 2011;121:4393-4408.

8. Ärnlöv J, Carlsson AC, Sundström J, et al. Serum FGF23 and risk of cardiovascular events in relation to mineral metabolism and cardiovascular pathology. Clin J Am Soc Nephrol 2013;8:781-786.

9. Arnlov J, Carlsson AC, Sundstrom J, et al. Higher fibroblast growth factor-23 increases the risk of all-cause and cardiovascular mortality in the community. Kidney Int 2013;83:160-166.

10. Parker BD, Schurgers LJ, Brandenburg VM, et al. The associations of fibroblast growth factor 23 and uncarboxylated matrix Gla protein with mortality in coronary artery disease: the Heart and Soul Study. Ann Intern Med 2010; 152:640-648.
11. Sacco RL, Boden-Albala B, Gan R, et al. Stroke incidence among white, black, and Hispanic residents of an urban community: the Northern Manhattan Stroke Study. Am J Epidemiol 1998;147:259-268.

12. Sacco RL, Boden-Albala B, Abel G, et al. Race-ethnic disparities in the impact of stroke risk factors: the Northern Manhattan Stroke Study. Stroke 2001;32:1725-1731.

13. Wallman KK, Hodgdon J. Race and ethnic standards for federal statistics and administrative reporting. Stat Rep 1977;77-110:450-454.

14. Gentry EM, Kalsbeek WD, Hogelin GC, et al. The behavioral risk factor surveys: II: design, methods, and estimates from combined state data. Am J Prev Med 1985;1:9-14.

15. Boden-Albala B, Cammack S, Chong J, et al. Diabetes, fasting glucose levels, and risk of ischemic stroke and vascular events: findings from the Northern Manhattan Study (NOMAS). Diabetes Care 2008;31:1132-1137.

16. Levey A, Kusek J, Beck G. A simplified equation to predict glomerular filtration rate from serum creatinine. J Am Soc Nephrol 2000;11:155A. Abstract.

17. Levey AS, Stevens LA, Schmid CH, et al. A new equation to estimate glomerular filtration rate. Ann Intern Med 2009;150:604-612.

18. Inker LA, Schmid CH, Tighiouart $\mathrm{H}$, et al. Estimating glomerular filtration rate from serum creatinine and cystatin C. N Engl J Med 2012;367:20-29.

19. Yilmaz MI, Sonmez A, Saglam M, et al. Longitudinal analysis of vascular function and biomarkers of metabolic bone disorders before and after renal transplantation. Am J Nephrol 2013;37:126-134.

20. Desjardins L, Liabeuf S, Renard C, et al. FGF23 is independently associated with vascular calcification but not bone mineral density in patients at various CKD stages. Osteoporos Int 2012;23:2017-2025.

21. Nakayama M, Kaizu Y, Nagata M, et al. Fibroblast growth factor 23 is associated with carotid artery calcification in chronic kidney disease patients not undergoing dialysis: a cross-sectional study. BMC Nephrol 2013;14:22.

22. Mirza MA, Larsson A, Melhus H, Lind L, Larsson TE. Serum intact FGF23 associate with left ventricular mass, hypertrophy and geometry in an elderly population. Atherosclerosis 2009;207:546-551.

23. van Ballegooijen AJ, Visser M, Cotch MF, et al. Serum vitamin $\mathrm{D}$ and parathyroid hormone in relation to cardiac structure and function: the ICELAND-MI substudy of AGESReykjavik. J Clin Endocrinol Metab 2013;98:2544-2552.

24. Piovesan A, Molineri N, Casasso F, et al. Left ventricular hypertrophy in primary hyperparathyroidism: effects of successful parathyroidectomy. Clin Endocrinol 1999;50:321-328.

25. Wermers RA, Khosla S, Atkinson EJ, et al. Incidence of primary hyperparathyroidism in Rochester, Minnesota, 1993-2001: an update on the changing epidemiology of the disease. J Bone Miner Res 2006;21:171-177.

26. Isakova $\mathrm{T}$, Xie $\mathrm{H}, \mathrm{Barchi-Chung} \mathrm{A}$, et al. Daily variability in mineral metabolites in CKD and effects of dietary calcium and calcitriol. Clin J Am Soc Nephrol 2012;7:820-828.

27. Brondum-Jacobsen P, Nordestgaard BG, Schnohr P, Benn M. 25-Hydroxyvitamin D and symptomatic ischemic stroke: an original study and meta-analysis. Ann Neurol 2013;73:38-47.

28. Hasegawa H, Nagano N, Urakawa I, et al. Direct evidence for a causative role of FGF23 in the abnormal renal phosphate handling and vitamin $\mathrm{D}$ metabolism in rats with early-stage chronic kidney disease. Kidney Int 2010;78:975-980. 